\title{
Neonatal meningitis in England and Wales: 10 years on
}

\author{
D E Holt, S Halket, J de Louvois, D Harvey
}

\begin{abstract}
Objectives-To determine the incidence of neonatal meningitis in England and Wales. Design-A national postal survey using the British Paediatric Surveillance Unit (BPSU) card scheme supplemented by information from other sources.

Setting-England and Wales 1996-1997.

Subjects-A total of 274 babies less than 28 days of age who were treated for meningitis.

Results-The incidence of neonatal meningitis in England and Wales has not changed since our previous study in 19851987. However, the acute phase mortality has fallen from $19.8 \%$ in $1985-1987$ to $6.6 \%$ in this study. Group B streptococci (42\%) and Escherichia coli (16\%) remain the most common infecting microorganisms. Eight of $69(12 \%)$ babies with group B streptococci and 4/26 (15\%) with $E$ coli died. Antibiotic regimens based on the third generation cephalosporins, notably cefotaxime, were most commonly used $(84 \%)$. The BPSU scheme identified $72 \%$ of cases during the study period. Most cases of viral meningitis were not reported through the BPSU. Less than a third of samples from aseptic meningitis were examined for viruses; $56 \%$ of these were positive.
\end{abstract}

Conclusions-Although the incidence of neonatal meningitis remains unchanged, mortality from this infection has fallen significantly. If this improvement is maintained as reflected in the level of sequelae at 5 years of age, then the fear surrounding meningitis during the neonatal period will have been dramatically reduced.

(Arch Dis Child Fetal Neonatal Ed 2001;84:F85-F89)

The Karim Centre for Meningitis Research, Imperial College School of Medicine, Department of Paediatrics and Neonatal Medicine, Queen Charlotte's and Chelsea Hospital, Goldhawk Road, London W6 0XG, UK

D E Holt

S Halket,

J de Louvois

D Harvey

Correspondence to: Professor Harvey,

Department of Paediatrics,

Imperial College School of

Medicine, Hammersmith

Hospital, Ducane Road,

London W12 0NN, UK

david.harvey@ic.ac.uk

Accepted 12 October 2000
Keywords: meningitis; group B streptococci; Escherichia coli; cephalosporins; viral meningitis; mortality

The study of infantile and neonatal meningitis carried out 13 years ago by de Louvois et $a l^{1}$ remains the only nationwide survey of the disease in this population group. In that prospective postal survey of paediatricians in England and Wales, carried out between 1985 and 1987, the incidence of neonatal meningitis from all causes was 0.32 per 1000 births with an incidence of bacterial meningitis of 0.22 per 1000. ${ }^{1}$ A review of national data for England and Wales for the years 1975-1991 collected by the Public Health Laboratory Service, Communicable Diseases Surveillance Unit (CDSC) showed that the mean annual total of laboratory reported cases of neonatal bacterial meningitis was 109 (range 69-133). ${ }^{2}$ Taking into account the falling birth rate over this period, this gave an annual incidence of $0.17 / 1000$ live births. A 10 year retrospective study between 1980 and 1989 of children resident in the Nottingham District Health Authority area showed an annual incidence of neonatal meningitis from all causes of 0.37 per 1000 births. $^{3}$ There is thus little evidence that the incidence of the disease has changed in the past 30 years even though there have been major changes to the neonatal population during this time. More recently there have been changes in the diagnosis, treatment, and supportive care of neonates with meningitis. ${ }^{4}$ To determine whether these changes have influenced the incidence, mortality, or management of neonatal meningitis during the 10 years since the first national survey, we set up a collaborative study between the Karim Centre for Meningitis Research and the British Paediatric Surveillance Unit (BPSU) which covered the United Kingdom and Republic of Ireland. The study protocol also included a commitment to the long term follow up of the patient group in order to determine the prevalence of serious sequelae.

\section{Subjects and methods}

STUDY ORGANISATION

Data were collected prospectively using the BPSU system of case notification by "orange cards" over an 18 month period from 1 July 1996 to 31 December 1997. During this time the monthly cards sent by the BPSU to about 1800 participants in the scheme included reference to cases of neonatal meningitis. Respondents were asked to report to the BPSU, in the usual way, all cases of meningitis that they had managed in the previous month. At the time of the study, the BPSU list included respondents in England, Wales, Scotland, Northern Ireland, and the Republic of Ireland. The list included all members of the Royal College of Paediatrics and Child Health, the Faculty of Paediatrics of the Royal College of Physicians of Ireland, and other non-College members in specialty groups. Thus all senior physicians likely to treat children were included. Compliance rates for return of the orange cards to the BPSU for the study period was $94 \%{ }^{6}$ When a case of neonatal meningitis was notified, standardised clinical and microbiological questionnaires were sent to the paediatrician and microbiologist involved in managing the case. To determine the efficiency of the BPSU scheme, additional information was collected from hospital microbiology laboratories, the PHLS Meningococcus Reference 
Laboratory in Manchester (MRL), the Office of National Statistics (ONS), and CDSC. Cases identified through these sources were only included if they had not been notified through the BPSU scheme.

\section{INCLUSION CRITERIA}

Paediatricians were asked to notify cases presenting as clinical meningitis in newborn babies of 28 days of age or less. This included cases where the diagnosis was presumptivethat is, based on clinical signs and not proven subsequently by cerebrospinal fluid (CSF) analysis and culture. Cases where meningitis was diagnosed at autopsy were included, but data on babies with neural tube defects or ventricular shunt who contracted meningitis were excluded.

DATA ANALYSIS

Data handling and statistical analyses were carried out using Epi Info (version 6) from the Division of Surveillance and Epidemiology, Epidemiology Program Office, Center for Disease Control and Prevention, Atlanta, GA 30333, USA.

\section{Results}

The overall response rate to the BPSU surveillance scheme was $94 \% .{ }^{6}$ During the 18 month study period, 282 cases of meningitis in newborn babies were identified in England and Wales, 18 in Scotland, 13 in Northern Ireland, and 14 in the Irish Republic. The response from paediatricians in Scotland, Northern Ireland, and the Republic of Ireland was such that the data could not be considered as representative, and these data were therefore excluded from the analysis. The results presented here are those from England and Wales. In the 282 cases identified in England and Wales, bacteria were reported in the CSF of 144, viruses in 18, fungi in four, and in 96 no organism was isolated, although every case presented clinically as meningitis and was managed accordingly. In 12 cases, diagnosis was based on clinical signs only, because for various clinical reasons a CSF was not collected. The remaining eight cases reported through the BPSU were not confirmed because, despite considerable effort, corroborative information from the paediatricians and microbiologists concerned was not forthcoming. In the absence of any information to confirm a diagnosis of meningitis, these eight cases were excluded from further consideration. A further 99 cases that had not been reported through the BPSU scheme were identified through hospital microbiology laboratories (five bacterial, one viral), MRL (seven bacterial), ONS (eight bacterial, eight unknown), and CDSC (40 bacterial, 29 viral, one fungal). Thus the study identified 373 cases of neonatal meningitis in England and Wales during the 18 month study period, and $73 \%(274 / 373)$ of these were reported through the BPSU scheme. The mean birth rate in England and Wales during the study period was 650800 per annum, giving an annual incidence of neonatal meningitis from
Table 1 Clinical details at diagnosis on 274 babies with meningitis

\begin{tabular}{lc}
\hline & Number of cases (\%) \\
\hline At diagnosis & \\
In coma & $17(6)$ \\
Convulsions & $57(21)$ \\
Rash & $8(3)$ \\
Antibiotic treatment in previous $48 \mathrm{~h}$ & $79(29)$ \\
Congenital anomaly & $7(3)$ \\
Hydrocephalus & $1(<1)$ \\
Previous surgery & $1(<1)$ \\
Less than 33 weeks gestation & $41(15)$ \\
Less than 2000 g birth weight & $46(17)$ \\
\hline
\end{tabular}

all causes of $0.39 / 1000$ births. The annual incidence of bacterial meningitis was $0.21 / 1000$ births and of viral meningitis $0.05 / 1000$. The 99 cases identified from sources other than the BPSU scheme are included in the calculation of incidence but, because full clinical and microbiological data could not be obtained, they are excluded from any further analysis. The results presented below relate only to the 274 cases notified through the BPSU. The proposed long term follow up will also be confined to these cases.

The male to female ratio for the 274 babies was $1.2: 1$, the mean (SD) birth weight was 2876 (901) g, and the mean (SD) gestational age 37 (4) weeks. A total of 127 babies, aged between 2 and 28 days, were admitted from home, and 121 were in hospital at the time of diagnosis (the route to hospital was not recorded in 26 cases); 25 babies were transferred from one hospital to another because of their meningitis. The mean age at diagnosis for the domiciliary admissions was 13 days compared with 7 days for those in hospital. Forty one $(15 \%)$ of the babies were born before 33 weeks gestation, and $46(17 \%)$ weighed less than $2000 \mathrm{~g}$ at birth. Among babies born in England and Wales in 1996, the incidence of a birth weight of less than $2000 \mathrm{~g}$ was $2.8 \% .^{7}$ Thirty seven (14\%) babies were born after premature rupture of membranes of 25 hours or more duration, and 16 babies (6\%) were born to mothers who were pyrexial. Meningitis within the first 10 days of life occurred in 153 babies $(56 \%)$ of the study group.

Lumbar puncture was performed on 263/ 274 babies $(96 \%)$. Pleocytosis of greater than $100 \times 10^{6}$ white blood cells/ 1 occurred in the CSF of 203 babies (74\%). Sixteen of 20 babies with less than $10 \times 10^{6}$ white blood cells/1 had positive cultures, 10 with bacteria (including

Table 2 Causative organisms in 274 cases of neonatal meningitis

\begin{tabular}{lll}
\hline & $\begin{array}{l}\text { Number of } \\
\text { cases }\end{array}$ & Died \\
\hline Group B $\beta$ haemolytic streptococci & $69(25)$ & $8(12)$ \\
Escherichia coli & $26(10)$ & $4(15)$ \\
Listeria monocytogenes & $7(3)$ & 1 \\
Haemophilus influenzae & $1(<1)$ & 0 \\
Neisseria meningitidis & $5(2)$ & 0 \\
Streptococcus pneumoniae & $8(3)$ & 1 \\
Other Gram positive bacteria & $17(6)$ & 0 \\
Other Gram negative bacteria & $11(4)$ & 0 \\
Enteroviruses & $18(7)$ & 0 \\
Candida albicans & $4(2)$ & 0 \\
Sterile CSF & $96(35)$ & $2(2)$ \\
CSF sample not collected & $12(4)$ & 2 \\
\hline
\end{tabular}

Values in parentheses are percentages. CSF, Cerebrospinal fluid. 
Table 3 Cause of neonatal meningitis according to whether babies were admitted to special baby care units from hospital or from home

\begin{tabular}{llll}
\hline & $\begin{array}{l}\text { Home admissions } \\
(n=127)\end{array}$ & $\begin{array}{l}\text { Hospital admissions } \\
(n=121)\end{array}$ & $\begin{array}{l}\text { Unknown route } \\
(n=26)\end{array}$ \\
\hline Group B streptococcus & $35(3)$ & $29(4)$ & $5(1)$ \\
Escherichia coli & 11 & $12(2)$ & $3(2)$ \\
Listeria monocytogenes & 5 & $2(1)$ & 0 \\
Streptococcus pneumoniae & $7(1)$ & 1 & 0 \\
Neisseria meningitidis & 5 & 1 & 0 \\
Haemophilus influenzae & 1 & 0 & 0 \\
Salmonella virchow & 1 & 0 & 0 \\
Moraxella catarrhalis & 1 & 1 (Strmitis) & 0 \\
a-haemolytic streptococcus & 1 & 1 & 0 \\
Staphylococcus epidermidis & 1 & 1 & 1 \\
Streptococcus faecalis & 0 & 1 & 0 \\
Staphylococcus aureus & 0 & 6 & 0 \\
Misc. Gram negative rods & 0 & 6 & 0 \\
Gram positive cocci & 4 & 0 & 0 \\
Gram negative cocci & 1 & 5 & 2 \\
Viruses & 11 & 4 & 0 \\
Candida sp & 0 & $51(2)$ & 14 \\
No organism isolated & $43(2)$ & &
\end{tabular}

Values in parentheses are numbers of deaths.

${ }^{\star}$ In Gram film of CSF.

three group B streptococci, two Escherichia coli, two Streptococcus pneumoniae), two with Candida sp, and four with viruses. Three babies with negative CSF cultures and low initial CSF white cell counts had counts in excess of $100 \times$ $10^{6}$ white blood cells $/ 1$ in a subsequent sample. Table 1 shows the clinical details at the time of diagnosis.

Table 2 shows the microorganisms isolated from CSF. Lancefield group B $\beta$ haemolytic streptococci and $E$ coli were the commonest causes, accounting for $25 \%$ and $10 \%$ of all cases, and $42 \%$ and $16 \%$ of culture positive cases respectively. Eleven of the enteroviruses isolated were identified as Coxsackie virus. Of the 114 cases of apparent aseptic meningitis, viral investigations were carried out on 32 (28\%) of the CSF samples. They were positive in $18(56 \%)$ of these. There were 108 babies that had negative cultures from their CSF (96) or had no CSF collected (12). Thirty four $(31 \%)$ of these 108 babies had positive blood cultures and significant organisms were isolated from other sites from a further 20 babies. All but one of 96 babies with negative CSF cultures had CSF white cell counts of $\geqslant 20 \times$ $10^{6} / 1$; in 66 samples the count exceeded $100 \times$ $10^{6} / 1$. The remaining one baby had a bulging fontanelle and group B streptococci in the eyes. Twenty seven (28\%) of the 96 babies with culture negative CSF had received antibiotics during the preceding 48 hours. In addition, 69/110 babies with a positive CSF culture also had a positive blood culture with the same organism. There were differences in the microbial isolates according to whether the baby was at home or in hospital before diagnosis (table 3). These differences may reflect the differences in postnatal age between the two populations.

The overall case fatality rate was $18 / 274$ $(6.6 \%)$. The mortality among babies with $E$ coli or group B streptococcal meningitis was $15 \%$ and $12 \%$ respectively. Single deaths were associated with meningitis due to Listeria monocytogenes and Str pneumoniae. In the group from which no CSF was collected, two of 12 died. Two babies with aseptic meningitis (2/96) also died. They had positive blood cultures with either $E$ coli or group B streptococci. There were no deaths in the group of babies suffering from viral meningitis. Mortality (6/17) was associated with the presence of coma on admission (odds ratio (OR) 11.14, 95\% confidence interval (CI) 3.01 to $40.87, \mathrm{p}=0.0003$ ). Mortality (5/23) was also higher among babies treated with steroids (OR 5.09, 95\% CI 1.38 to $17.94, p=0.01)$. Three of $79(4 \%)$ who received antibiotics during the 48 hours before diagnosis, died, compared with 9/146 (6.2\%) reported not to have received antibiotics. Mortality among babies admitted from home was similar (5\%) to that among babies diagnosed in the hospital where they were treated $(6 \%)$. It was higher (12\%) among babies transferred from one hospital to another for treatment. Two of the 18 babies who died were less than 33 weeks gestation, and five weighed less than $2000 \mathrm{~g}$ at birth. Thus mortality in these two groups was higher $(8.3 \%$ and $11 \%$ respectively) than in the population as a whole. Five of the 18 babies who died were less than 72 hours old. There was no significant difference in mortality for each of the four postnatal weeks, rates being $6.7 \%, 5.0 \%, 8.5 \%$, and $7.4 \%$ respectively.

Table 4 shows the antimicrobial agents used to treat babies in this study. Antibiotic regimens based on cefotaxime were used most commonly $(214 / 274 ; 78 \%)$ with other third generation cephalosporins (ceftazidime 10 and ceftriaxone 6) being occasionally used. In most cases, the third generation cephalosporin was combined with a penicillin or ampicillin compound, with or without an aminoglycoside. Thirty five babies received treatment with other regimens, notably penicillin/ampicillin with an aminoglycoside, usually gentamicin. Three babies received chloramphenicol together with other antibiotics. The four cases of Candida meningitis were treated with amphotericin with or without other antifungal drugs. Four babies with viral meningitis received acyclovir and 12 did not. Information on the remaining two cases was not available. Eight of the 96 babies with sterile CSF also received acyclovir. All the babies treated with acyclovir and all those with confirmed viral meningitis also received antibacterial agents. Four babies (three with $E$ coli infection and one with group B streptococci) died within 48 hours of the start of antibiotic treatment; all were more than

Table 4 Treatment of 274 newborn babies with meningitis

\begin{tabular}{lc}
\hline & $\begin{array}{c}\text { Number of babies receiving } \\
\text { each drug (\%) }\end{array}$ \\
\hline Cefotaxime & $214(78)$ \\
Ceftazadime & $11(4)$ \\
Other cephalosporins & $13(5)$ \\
Gentamicin & $123(45)$ \\
Other aminoglycosides & $12(4)$ \\
Penicillin & $105(38)$ \\
Ampicillin & $52(19)$ \\
Other penicillins & \\
$\quad$ Amoxycillin & $44(16)$ \\
$\quad$ Flucloxacillin & $22(8)$ \\
Chloramphenicol & $3(1)$ \\
Vancomycin & $13(5)$ \\
Other antibiotics & $29(13)$ \\
Acyclovir & $14(5)$ \\
Barbiturates & $35(13)$ \\
Steroids & $23(8)$ \\
\hline
\end{tabular}


Table 5 Mortality associated with complications following neonatal meningitis

\begin{tabular}{lc}
\hline & Percentage \\
\hline Mortality following any complication & $11.4(8 / 70)$ \\
Mortality following convulsions & $12.3(7 / 57)$ \\
Mortality following hydrocephalus & $4.5(1 / 22)$ \\
Mortality following relapse & $25.0(2 / 8)$ \\
Mortality among babies without complications & $4.9(10 / 204)$ \\
\hline
\end{tabular}

36 weeks gestation. Based on the results from the reporting laboratories, antibiotic resistance was shown in $30(11 \%)$ bacterial isolates, with resistance to ampicillin $(14 / 30,47 \%)$ being the most common.

Complications due to ventriculitis, hydrocephalus, convulsions, and relapse were reported in $70 / 274(26 \%)$ of babies. Complications were more common in babies with confirmed bacterial meningitis (51/144 $(35.4 \%))$ than in those infected with other microorganisms $(4 / 22(18 \%))$ or with negative or no CSF cultures $(15 / 108(14 \%))$. Ten of these 15 babies with negative or no CSF cultures had positive blood cultures. Only one baby suffered from herniation of the spinal cord (coning) after lumbar puncture. Mortality among babies with complications was higher $(11 \%$; table 5$)$ than in uncomplicated cases $(4.9 \%)$. The worst prognosis was in babies who suffered convulsions, where 7/57 (12\%) died. Eight babies were reported to have relapsed, and two of them died. Six of the eight were admitted from home, including the single case of Salmonella meningitis, due to Salmonella virchow. In none of the relapse cases had antibiotics apparently been given during the 48 hours before diagnosis, although the CSF was sterile in three, all of which had a pleocytosis.

\section{Discussion}

One of the major issues raised by our earlier study ${ }^{1}$ was that cases of meningitis in England and Wales were underreported, particularly those caused by viruses. The study reported here was carried out using the BPSU monthly reporting system. Of the 373 cases of neonatal meningitis known to have occurred in England and Wales during the study period, $27 \%$ were not reported through the BPSU route, which is considerably higher than the 5\% not reported through our own card system in 1985-1987. ${ }^{1}$ Therefore sole reliance on self reporting methods will result in underreporting and incomplete results unless data from other sources can be fully validated. ${ }^{8}$ The incidence of neonatal meningitis from all causes remains very similar $(0.39 / 1000)$ to that reported 13 years ago $(0.32 / 1000) .{ }^{1}$ The incidence of proven bacterial meningitis also shows no difference 13 years on (0.22/1000 then, 0.21/1000 now). Birth before 33 weeks gestation and a birth weight below $2000 \mathrm{~g}$ remain significant risk factors. Neonatal meningitis is six times more common in babies weighing less than $2000 \mathrm{~g}$ than in those above $2000 \mathrm{~g}$. The mortality in these two groups is also higher.

The overall death rate reported in this study, $6.6 \%$, is dramatically lower than the $19.8 \%$ mortality reported in the 1985-1987 study $^{1}$ $(\mathrm{p}<0.001)$. Among bacteriologically proven cases of meningitis, the mortality has also fallen, from $29 \%$ in the $1985-1987$ study to $10 \%(14 / 144)$ in this study (table 2). Thus mortality from neonatal meningitis appears to be falling. This is in contrast with childhood meningitis as a whole, for which there has been no significant decline in mortality despite the advent of new antimicrobial agents. ${ }^{9}$ Our own reanalysis of the 1985-1987 data in the light of subsequent follow up showed that use of third generation cephalosporins in the treatment of neonatal meningitis correlated with a decrease in mortality but not morbidity. ${ }^{10}$ The proposed follow up study of these children at 5 years of age will focus specifically on this question. The standard of neonatal intensive care has improved over the past decade, but it is not apparent from this study that treatment is being initiated earlier; of those babies admitted from home, $21 \%$ received antibiotics in the 48 hours preceding hospital admission (table 2) compared with $21 \%$ in the earlier study. ${ }^{1}$

Coma at diagnosis remains a significant risk factor for death as described in our earlier study. ${ }^{1}$ However, morbidity also remains high in babies treated with steroids. It was suggested by de Louvois et $a l^{1}$ that babies who received steroids were likely to be those that were most severely ill. While that supposition cannot be ruled out here, we have no direct evidence that this is the case. None was admitted in coma for example. There has been no increase in the use of steroids for the treatment of neonatal meningitis over the past 13 years, remaining at $8 \%$ of cases.

The prevalence of group B streptococci as the dominant cause of meningitis in the newborn continues to rise. In this study, it accounted for $48 \%$ of culture proven bacterial meningitis compared with $38 \%$ in the 19851987 study. ${ }^{1} E$ coli was for many years the commonest single cause of neonatal meningitis in the United Kingdom. ${ }^{11}$ However, since the 1980 s, the proportion of cases caused by $E$ coli has declined while that caused by group B streptococci has increased. The prevalence of $E$ coli meningitis has fallen from $25 \%$ in the $1985-1987$ study to $18 \%$ in this study. However, the relatively high death rate following meningitis caused by $E$ coli and other Gram negative enteric bacilli continues.

During the last 15 years, there have been dramatic changes in the antibiotic regimens used in the treatment of neonatal meningitis in the United Kingdom. Data from the 19851987 survey $^{1}$ showed that $55 \%$ of babies received regimens based on gentamicin and $48 \%$ regimens based on chloramphenicol, in both cases in combination with other antibiotics. A national survey in the early $1990 \mathrm{~s}^{4}$ showed that the situation was changing. At that time only $8 \%$ of paediatricians would have prescribed chloramphenicol, while $58 \%$ would have prescribed regimens based on the third generation cephalosporins, in line with the recommendations of the Committee on Infectious Diseases of the American Academy of Pediatrics. ${ }^{12}$ This trend has continued, and in this study $84 \%$ of babies received cephalosporin 
based regimens and most of the remainder received aminoglycoside based regimens.

Vancomycin is the treatment of choice for staphylococcal meningitis; ${ }^{13}$ however, in view of the infrequency of primary staphylococcal meningitis in the United Kingdom, it should only be given when staphylococcal infection has been confirmed by culture.

The true incidence of viral meningitis has remained elusive. Some $63 \%$ of cases of viral meningitis identified in this study were not notified through the BPSU scheme. The incidence of proven viral meningitis in 19851987 was $0.012 / 1000$ births, ${ }^{1}$ and this was recognised as an underestimate. ${ }^{114}$ The number of cases reported here (0.05/1000 live births) suggests that the incidence has increased; however, a number of other factors, including improved laboratory procedures, could account for the increase. Only 32 of $114 \mathrm{CSF}$ samples that had negative bacteriological cultures were examined for viruses. Eighteen $(56 \%)$ of these were positive.

It is disappointing that less than a third of the bacteriologically sterile CSF samples in this study were submitted to viral investigation given that, when these studies were carried out, more than half of the samples were positive. Viral studies should be part of the routine investigation of neonatal meningitis when culture has failed to yield a bacterial pathogen. Although there is currently no effective treatment for viral meningitis, a positive diagnosis provides considerable reassurance to the parents and may allow earlier discharge from the neonatal intensive care unit. It has been shown that patients benefit from identification of enterovirus as the cause of aseptic meningitis. ${ }^{15}$ Our results did not suggest any benefit in the acute phase from administering acyclovir to either the virus positive group or the bacteria negative group. These findings are in agreement with others. ${ }^{16}$ Whether the use of acyclovir helps to prevent long term sequelae has yet to be determined.
The results of this national survey, when compared with that carried out previously, show that, although the incidence of neonatal meningitis remains unchanged, the mortality has fallen significantly. If this improvement is also reflected in the level of sequelae when these children are assessed at 5 years of age, then the fear surrounding meningitis during the neonatal period will have been dramatically reduced.

We are grateful to the organisers of the BPSU scheme for their support, and to the paediatricians and microbiologists who provided us with information. We would also like to thank microbiological colleagues and the ONS, CDSC, and MRL for their help in tracing cases that were not identified through the BPSU scheme. We thank the Fayed Charitable Foundation for financial support.

1 de Louvois J, Blackbourn J, Hurley R, et al. Infantile meningitis in England and Wales: a two year study. Arch Dis Child 1991;66:603-7.

2 Synott MB, Morse DL, Hall SM. Neonatal meningitis in England and Wales: a review of routine national data. Arch Dis Child 1991;71:K75-80.

3 Fortnum HM, Davis AC. Epidemiology of bacterial meningitis. Arch Dis Child 1993;68:763-7.

4 Harvey D, Holt DE, Bedford H. Bacterial meningitis in the newborn: a prospective study of mortality and morbidity. newborn: a prospective study of
Semin Perinatol 1999;23:218-25.

5 Hall SM, Glickman M. The British Paediatric Surveillance Unit. Arch Dis Child 1988;63:344-6.

6 Guy M, Nicoll A, Lynn R, eds. British Paediatric Surveillance Unit. 12th Annual Report 1997-1998. London: British Paediatric Surveillance Unit, 1998.

7 Office of National Statistics. Annual Reference Volume DH3 1997 and DH3 Monitor 98/3. London: HMSO, August 1998.

8 Dezateux C, Godward S. Validation of the reporting bases of the orthopaedic and paediatric surveillance schemes. Arch Dis Child 1996;75:232-6.

9 Peltola H. Controversies in the management of childhood meningitis. 7 Med Microbiol 1997;46:901-2.

10 Harvey D, Holt D, Bedford H. Bacterial meningitis in the newborn: a prospective study of mortality and morbidity. Semin Neonatol 1999;23:218-25.

11 de Louvois J. Acute bacterial meningitis in the newborn. $f$ Antimicrob Chemother 1994;24(suppl A):61-73.

12 Plotkin SA, Daum RS, Giebink GS, et al. Treatment of bacPlotkin SA, Daum RS, Giebink GS, et al. Tre

13 Wubbel L, McCracken GH. Management of bacterial meningitis. Pediatr Rev 1998;19:78-84.

14 Davies PA, Rudd PT. Neonatal meningitis. Cambridge: Mac Keith Press, 1994.

5 Wildin S, Chonmaitree T. The importance of the virology laboratory in the diagnosis and management of viral meningitis. American fournal of Diseases in Children 1987;141:454-7.

16 Marshall GS, Hauck MA, Buck G, et al. Potential cost savings through rapid diagnosis of enteroviral meningitis. Pediatr Infect Dis ₹ 1997;16:1086-7. 